\title{
Comorbidity and its impact on 1590 patients with COVID-19 in China: a nationwide analysis
}

\author{
Wei-jie Guan ${ }^{1,26}$, Wen-hua Liang ${ }^{2,26}$, Yi Zhao ${ }^{2,26}$, Heng-rui Liang ${ }^{2,26}$,

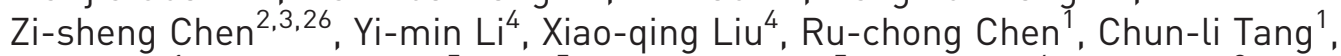 \\ Tao Wang ${ }^{1}$, Chun-quan Ou ${ }^{5}$, Li Li ${ }^{5}$, Ping-yan Chen ${ }^{5}$, Ling Sang ${ }^{4}$, Wei Wang ${ }^{2}$, \\ Jian-fu Li ${ }^{2}$, Cai-chen $\mathrm{Li}^{2}$, Li-min $\mathrm{Ou}^{2}$, Bo Cheng ${ }^{2}$, Shan Xiong ${ }^{2}$, Zheng-yi $\mathrm{Ni}^{6}$, \\ Jie Xiang ${ }^{6}$, Yu Hu ${ }^{7}$, Lei Liu ${ }^{8,9}$, Hong Shan ${ }^{10}$, Chun-liang Lei ${ }^{11}$, Yi-xiang Peng ${ }^{12}$, \\ $\mathrm{Li} \mathrm{Wei}^{13}$, Yong Liu ${ }^{14}$, Ya-hua Hu${ }^{15}$, Peng Peng ${ }^{16}$, Jian-ming Wang ${ }^{17}$, \\ Ji-yang Liu ${ }^{18}$, Zhong Chen ${ }^{19}$, Gang Li ${ }^{20}$, Zhi-jian Zheng ${ }^{21}$, Shao-qin Qiü ${ }^{22}$, \\ Jie Luo ${ }^{23}$, Chang-jiang Ye ${ }^{24}$, Shao-yong Zhu ${ }^{25}$, Lin-ling Cheng ${ }^{1}$, Feng $\mathrm{Ye}^{1}$, \\ Shi-yue $\mathrm{Li}^{1}$, Jin-ping Zheng ${ }^{1}$, Nuo-fu Zhang ${ }^{1}$, Nan-shan Zhong ${ }^{1}$ and \\ Jian-xing $\mathrm{He}^{2}$, on behalf of the China Medical Treatment Expert Group for \\ COVID-19
}

@ERSpublications

The presence and number of comorbidities predict clinical outcomes of COVID-19 http://bit.ly/3b9ibw5

Cite this article as: Guan W-j, Liang W-h, Zhao Y, et al. Comorbidity and its impact on 1590 patients with COVID-19 in China: a nationwide analysis. Eur Respir J 2020; 55: 2000547 [https://doi.org/10.1183/ 13993003.00547-2020].

\section{ABSTRACT}

Background: The coronavirus disease 2019 (COVID-19) outbreak is evolving rapidly worldwide.

Objective: To evaluate the risk of serious adverse outcomes in patients with COVID-19 by stratifying the comorbidity status.

Methods: We analysed data from 1590 laboratory confirmed hospitalised patients from 575 hospitals in 31 provinces/autonomous regions/provincial municipalities across mainland China between 11 December 2019 and 31 January 2020. We analysed the composite end-points, which consisted of admission to an intensive care unit, invasive ventilation or death. The risk of reaching the composite end-points was compared according to the presence and number of comorbidities.

Results: The mean age was 48.9 years and 686 (42.7\%) patients were female. Severe cases accounted for $16.0 \%$ of the study population. 131 (8.2\%) patients reached the composite end-points. 399 (25.1\%) reported having at least one comorbidity. The most prevalent comorbidity was hypertension (16.9\%), followed by diabetes (8.2\%). $130(8.2 \%)$ patients reported having two or more comorbidities. After adjusting for age and smoking status, COPD (HR (95\% CI) 2.681 (1.424-5.048)), diabetes (1.59 (1.03$2.45)$ ), hypertension (1.58 (1.07-2.32)) and malignancy (3.50 (1.60-7.64)) were risk factors of reaching the composite end-points. The hazard ratio (95\% CI) was 1.79 (1.16-2.77) among patients with at least one comorbidity and 2.59 (1.61-4.17) among patients with two or more comorbidities.

Conclusion: Among laboratory confirmed cases of COVID-19, patients with any comorbidity yielded poorer clinical outcomes than those without. A greater number of comorbidities also correlated with poorer clinical outcomes.

This article has supplementary material available from erj.ersjournals.com

Received: 04 March 2020 | Accepted after revision: 13 March 2020

Copyright CERS 2020. This version is distributed under the terms of the Creative Commons Attribution NonCommercial Licence 4.0. 


\section{Introduction}

Since November 2019, the rapid outbreak of coronavirus disease 2019 (COVID-19), which arose from severe acute respiratory syndrome coronavirus 2 (SARS-CoV-2) infection, has recently become a public health emergency of international concern [1]. COVID-19 has contributed to an enormous adverse impact globally. As of 10 March 2020 there have been 113702 laboratory confirmed cases and 4012 deaths globally [2].

According to the latest reports, the clinical manifestations of COVID-19 are heterogeneous [3-12]. On admission, $20-51 \%$ of patients were reported as having at least one comorbidity, with diabetes (10-20\%), hypertension (10-15\%) and other cardiovascular and cerebrovascular diseases (7-40\%) being most common $[3,4,6]$. Previous studies have demonstrated that the presence of any comorbidity has been associated with a 3.4-fold increased risk of developing acute respiratory distress syndrome in patients with H7N9 infection [13]. As with influenza [14-18], SARS-CoV [19] and Middle East Respiratory Syndrome coronavirus (MERS-CoV) [20-28], COVID-19 is more readily predisposed to respiratory failure and death in susceptible patients $[4,5]$. Nonetheless, previous studies have had certain limitations in study design including the relatively small sample sizes and single centre observations. Studies that address these limitations are needed to explore the factors underlying the adverse impact of COVID- 19.

Our objective was to evaluate the risk of serious adverse outcomes in patients with COVID-19 by stratification according to the number and type of comorbidities, thus unravelling the sub-populations with poorer prognosis.

\section{Methods}

Data sources and data extraction

This was a retrospective case study that collected data from patients with COVID-19 throughout China, under the coordination of the National Health Commission which mandated the reporting of clinical information from individual designated hospitals that admitted patients with COVID-19. This study is approved by the ethics committee of the First Affiliated Hospital of Guangzhou Medical University. After careful review of medical charts, we compiled the clinical data of laboratory confirmed hospitalised cases from 575 hospitals (representing 31.7\% of the certified hospitals admitting patients with COVID-19) between 11 December 2019 and 31 January 2020. The diagnosis of COVID-19 was made based on the World Health Organization interim guidance [29]. Because of the urgency of data extraction, complete random sampling could not be applied in our settings. All clinical profiles outside the Hubei province were centrally provided by the National Health Commission. Three respiratory experts from Guangzhou were dispatched to Wuhan for raw data extraction from Wuhan JinYinTan Hospital where most cases in Wuhan were located. Our cohort included 132 patients from Wuhan JinYinTan Hospital, and one each

Affiliations: 'State Key Laboratory of Respiratory Disease and National Clinical Research Center for Respiratory Disease, the First Affiliated Hospital of Guangzhou Medical University, Guangzhou Medical University, Guangzhou, China. ${ }^{2}$ Dept of Thoracic Oncology and Surgery, China State Key Laboratory of Respiratory Disease and National Clinical Research Center for Respiratory Disease, the First Affiliated Hospital of Guangzhou Medical University, Guangzhou, China. ${ }^{3}$ The sixth Affiliated Hospital of Guangzhou Medical University, Qingyuan, China. ${ }^{4}$ Dept of Pulmonary and Critical Care Medicine, China State Key Laboratory of Respiratory Disease and National Clinical Research Center for Respiratory Disease, the First Affiliated Hospital of Guangzhou Medical University, Guangzhou, China. ${ }^{5}$ State Key Laboratory of Organ Failure Research, Dept of Biostatistics, Guangdong Provincial Key Laboratory of Tropical Disease Research, School of Public Health, Southern Medical University, Guangzhou, China. ${ }^{6}$ Wuhan Jin-yintan Hospital, Wuhan, China. ${ }^{7}$ Union Hospital, Tongji Medical College, Huazhong University of Science and Technology, Wuhan, China. ${ }^{8}$ Shenzhen Third People's Hospital, Shenzhen, China. ${ }^{9}$ The Second Affiliated Hospital of Southern University of Science and Technology, National Clinical Research Center for Infectious Diseases, Shenzhen, China. ${ }^{10}$ The Fifth Affiliated Hospital of Sun Yat-sen University, Zhuhai, China. ${ }^{11}$ Guangzhou Eighth People's Hospital, Guangzhou Medical University, Guangzhou, China. ${ }^{12}$ The Central Hospital of Wuhan, Wuhan, China. ${ }^{13}$ Wuhan No. 1 Hospital, Wuhan Hospital of Traditional Chinese and Western Medicine, Wuhan, China. ${ }^{14}$ Chengdu Public Health Clinical Medical Center, Chengdu, China. ${ }^{15}$ Huangshi Central Hospital of Edong Healthcare Group, Affiliated Hospital of Hubei Polytechnic University, Huangshi, China. ${ }^{16}$ Wuhan Pulmonary Hospital, Wuhan, China. ${ }^{17}$ Tianyou Hospital Affiliated to Wuhan University of Science and Technology, Wuhan, China. ${ }^{18}$ The First Hospital of Changsha, Changsha, China. ${ }^{19}$ The Third People's Hospital of Hainan Province, Sanya, China. ${ }^{20}$ Huanggang Central Hospital, Huanggang, China. ${ }^{21}$ Wenling First People's Hospital, Wenling, China.

${ }^{22}$ The Third People's Hospital of Yichang, Yichang. China. ${ }^{23}$ Affiliated Taihe Hospital of Hubei University of Medicine, Shiyan, China. ${ }^{24}$ Xiantao First People's Hospital, Xiantao, China. ${ }^{25}$ The People's Hospital of Huangpi District, Wuhan, China. ${ }^{26}$ These authors are joint first authors.

Correspondence: Jian-xing He, Dept of Thoracic Surgery, the First Affiliated Hospital of Guangzhou Medical University; China State Key Laboratory of Respiratory Disease and National Clinical Research Center for Respiratory Disease, Guangzhou, China. E-mail: drjianxing.helagmail.com; Nan-Shan Zhong, State Key Laboratory of Respiratory Disease, National Clinical Research Center for Respiratory Disease, Guangzhou Institute of Respiratory Health, The First Affiliated Hospital of Guangzhou Medical University, 151 Yanjiang Road, Guangzhou, China. E-mail: nanshandvip.163.com 
from 338 hospitals. Our cohort represented the overall situation as of 31 January 2020, taking into account the proportion of hospitals $(\sim 25 \%)$ and patient number $(1590(13.5 \%)$ out of 11791 cases), as well as the broad coverage (covering all major provinces/cities/autonomous regions). Confirmed cases denoted the patients whose high throughput sequencing or real-time, reverse-transcription PCR assay findings for nasal and pharyngeal swab specimens were positive [3]. For further details refer to the supplementary material. The interval between the potential earliest date of transmission source contacts (wildlife, suspected or confirmed cases) and the potential earliest date of symptom onset (i.e. cough, fever, fatigue and myalgia) was used to calculate the incubation period. Because the latest date was recorded in some patients who had continuous exposure to contamination sources, the incubation period of $<1$ day was not included in our analysis. The incubation periods were summarised based on the patients who had delineated the specific date of exposure.

The clinical data (including recent exposure history, clinical symptoms and signs, comorbidities, and laboratory findings upon admission) were reviewed and extracted by experienced respiratory clinicians, who subsequently entered the data into a computerised database for further double checking of all cases. Manifestations on chest radiograph or computed tomography (CT) was summarised by integrating the documentation or description in medical charts and, if available, a further review by our medical staff. Major disagreement of the radiologic manifestations between the two reviewers was resolved by consultation with another independent reviewer. Because the disease severity reportedly predicted poorer clinical outcomes of avian influenza [13], patients were classified as having severe or non-severe COVID-19 based on the 2007 American Thoracic Society Infectious Disease Society of America guidelines [30], taking into account its global acceptance for severity stratification of community-acquired pneumonia although no validation was conducted in patients with viral pneumonia. The predictive ability of the need for intensive care unit (ICU) admission and mortality has been validated previously [31, 32]. Briefly, severe cases denoted at least one major criterion (septic shock requiring vasoactive medications, or respiratory failure requiring mechanical ventilation), or at least three minor criteria (respiratory rate $\geqslant 30 \mathrm{breaths} \cdot \mathrm{min}^{-1}$, oxygen index $\leqslant 250$, multiple lobe infiltration, delirium or loss of consciousness, blood urea nitrogen $\geqslant 20 \mathrm{mg} \cdot \mathrm{dL}^{-1}$, blood leukocyte count $\leqslant 4000$ cells $\cdot \mathrm{dL}^{-1}$, blood platelet count $\leqslant 100000 \mathrm{cells} \cdot \mathrm{dL}^{-1}$, body temperature $<36^{\circ} \mathrm{C}$, and hypotension necessitating vasoactive drugs for maintaining blood pressure).

Comorbidities were determined based on patient's self-report on admission. Comorbidities were initially treated as a categorical variable (yes versus no) and subsequently classified based on the number (single versus multiple). Furthermore, comorbidities were sorted according to the organ systems (i.e. respiratory, cardiovascular, endocrine). Comorbidities that were classified into the same organ system (i.e. coronary heart disease, hypertension) would be merged into a single category.

The primary end-point of our study was a composite measure which consisted of the admission to ICU, invasive ventilation or death. This composite measure was adopted because all individual components were serious outcomes of H7N9 infections [13]. The secondary end-point was the mortality rate.

\section{Statistical analysis}

Statistical analyses were conducted with SPSS software (version 23.0; SPSS, Chicago, IL, USA). No formal sample size estimation was made because there has not been any published nationwide data on COVID-19. Nonetheless, our sample size was deemed sufficient to power the statistical analysis given its representativeness of the national patient population. Continuous variables were presented as mean $\pm \mathrm{SD}$ or median (interquartile ranges (IQR)) as appropriate, and the categorical variables were presented as counts and percentages. Since no random sampling was conducted, all statistical analyses were descriptive and no p-values were presented for the statistical comparisons except for the Cox proportional hazards regression model. Cox proportional hazards regression models were applied to determine the potential risk factors associated with the composite end-points, with the hazard ratio and 95\% confidence interval being reported. Our findings indicated that the statistical assumption of proportional hazards analysis was not violated. Moreover, a Cox regression model was considered more appropriate than a logistic regression model because it took into account the potential impact of the various durations of follow-up from individual patients. Age and smoking status were adjusted for in the proportional hazards regression model because they had been recognised as the risk factors of comorbidities even in the general population. Smoking status was stratified as current smoker, ex-smoker and never-smoker in the regression models.

\section{Results}

\section{Demographic and clinical characteristics}

The National Health Commission diagnosed 11791 patients in China with laboratory confirmed COVID-19 as of 31 January 2020. At this time-point, for data cut-off, our database included 1590 cases from 575 hospitals in 31 province/autonomous regions/provincial municipalities (refer to supplementary 
material). Of these 1590 cases, mean age was 48.9 years. 686 (42.7\%) patients were female. 647 (40.7\%) patients were managed inside the Hubei province, and 1334 (83.9\%) patients had contact history with Wuhan city. The most common symptom was fever on or after hospitalisation (88.0\%), followed by dry cough $(70.2 \%)$. Fatigue $(42.8 \%)$ and productive cough $(36.0 \%)$ were less common. At least one abnormal chest CT manifestation (including ground-glass opacities, pulmonary infiltrates and interstitial disorders) was identified in $>70 \%$ of patients. Severe cases accounted for $16.0 \%$ of the study population. $131(8.2 \%)$ patients reached the composite end-points during the study (table 1). Overall, the median (IQR) follow-up duration was 10 (8-14) days.

\section{Presence of comorbidities and clinical characteristics and outcomes of COVID-19}

Of the 1590 cases, 399 (25.1\%) reported having at least one comorbidity. The prevalence of specific comorbidities was: hypertension $(n=269,16.9 \%)$, other cardiovascular diseases $(n=59,3.7 \%)$ cerebrovascular diseases $(n=30,1.9 \%)$, diabetes $(n=30,8.2 \%)$, hepatitis B infections $(n=28,1.8 \%)$, COPD $(\mathrm{n}=24,1.5 \%)$, chronic kidney diseases $(\mathrm{n}=21,1.3 \%)$, malignancy $(\mathrm{n}=18,1.1 \%)$ and immunodeficiency $(n=3,0.2 \%)$. None of the cases had doctor-diagnosed asthma. At least one comorbidity was seen more commonly in severe cases than in non-severe cases (32.8\% versus $10.3 \%)$. Patients with at least one comorbidity were older (mean age 60.8 versus 44.8 years), were more likely to have shortness of breath ( $41.4 \%$ versus $17.8 \%$ ), nausea or vomiting ( $10.4 \%$ versus $4.3 \%)$, and tended to have abnormal chest radiograph manifestations $(29.2 \%$ versus $15.1 \%)$ (table 1$)$.

\section{Clinical characteristics and outcomes of COVID-19 stratified by the number of comorbidities}

We have further identified 130 (8.2\%) patients who reported having two or more comorbidities. Two or more comorbidities were more commonly seen in severe cases than in non-severe cases $(40.0 \%$ versus 29.4\%). Patients with two or more comorbidities were older (mean age 66.2 versus 58.2 years), were more likely to have shortness of breath (55.4\% versus $34.1 \%)$, nausea or vomiting ( $11.8 \%$ versus $9.7 \%$ ), unconsciousness (5.1\% versus $1.3 \%$ ) and have less abnormal chest radiographs (20.8\% versus $23.4 \%$ ) compared with patients who had one comorbidity (table 2).

Clinical characteristics and outcomes of COVID-19 stratified by organ systems of comorbidities A total of $269(16.9 \%)$ patients reported hypertension, 59 (3.7\%) reported cardiovascular diseases, 30 (1.9\%) reported cerebrovascular diseases, 30 (8.2\%) reported diabetes, 28 (1.8\%) reported hepatitis B infections, $24(1.5 \%)$ reported COPD, 21 (1.3\%) reported chronic kidney diseases, 18 (1.1\%) reported malignancy and three $(0.2 \%)$ reported immunodeficiency. Severe cases were more likely to have hypertension (32.7\% versus $12.6 \%$ ), cardiovascular diseases (33.9\% versus $15.3 \%)$, cerebrovascular diseases ( $50.0 \%$ versus $15.3 \%$ ), diabetes (34.6\% versus $14.3 \%)$, hepatitis B infections ( $32.1 \%$ versus $15.7 \%)$, COPD (62.5\% versus $15.3 \%)$, chronic kidney diseases (38.1\% versus $15.7 \%$ ) and malignancy (50.0\% versus $15.6 \%)$ compared with non-severe cases. Furthermore, comorbidities were more common in patients treated in the Hubei province compared with those managed outside the Hubei province as well as patients with a Wuhan exposure history compared with those without (table 3).

\section{Prognostic analyses}

Overall, 131 (8.3\%) patients reached the composite end-points during the study. 50 (3.1\%) patients died, $99(6.2 \%)$ were admitted to the ICU and $50(3.1 \%)$ received invasive ventilation. The composite end-point was documented in $77(19.3 \%)$ patients who had at least one comorbidity as opposed to 54 (4.5\%) patients without comorbidities. 37 (28.5\%) patients had two or more comorbidities. Significantly more patients with hypertension ( $19.7 \%$ versus $5.9 \%)$, cardiovascular diseases $(22.0 \%$ versus $7.7 \%)$, cerebrovascular diseases $(33.3 \%$ versus $7.8 \%$ ), diabetes (23.8\% versus $6.8 \%$ ), COPD (50.0\% versus $7.6 \%$ ), chronic kidney diseases $(28.6 \%$ versus $8.0 \%)$ and malignancy (38.9\% versus $7.9 \%)$ reached the composite end-points compared with those without (table 3).

Patients with two or more comorbidities had significantly escalated risks of reaching to the composite end-point compared with those who had a single comorbidity, and even more so compared with those with no comorbidities (all $\mathrm{p}<0.05$ ) (figure 1). After adjusting for age and smoking status, patients with COPD (HR (95\% CI) $2.68(1.42-5.05)$ ), diabetes (1.59 (1.03-2.45)), hypertension (1.58 (1.07-2.32)) and malignancy $(3.50(1.60-7.64))$ were more likely to reach the composite end-points than those without (figure 2). Results of unadjusted analysis are presented in table E1 and E2. Overall, findings of unadjusted and adjusted analysis were not materially altered. As compared with patients without comorbidity, the HR (95\% CI) was 1.79 (1.16-2.77) among patients with at least one comorbidity and 2.59 (1.61-4.17) among patients with two or more comorbidities (figure 2). Subgroup analysis by stratifying patients according to their age ( $<65$ years versus $\geqslant 65$ years) did not reveal substantial difference in the strength of associations between the number of comorbidities and mortality of COVID-19 (table E3). 
TABLE 1 Demographics and clinical characteristics of patients with or without any comorbidities

\begin{tabular}{|c|c|c|c|}
\hline \multirow[t]{2}{*}{ Variables } & \multicolumn{3}{|c|}{ Any comorbidity } \\
\hline & Total & No & Yes \\
\hline Subjects $n$ & 1590 & 1191 & 399 \\
\hline Age years & $48.9 \pm 16.3$ & $44.8 \pm 15.2$ & $60.8 \pm 13.4$ \\
\hline Incubation period day & $3.6 \pm 4.2$ & $3.7 \pm 4.3$ & $3.5 \pm 3.9$ \\
\hline Temperature on admission ${ }^{\circ} \mathrm{C}$ & $37.4 \pm 0.9$ & $37.4 \pm 0.9$ & $37.3 \pm 0.9$ \\
\hline Respiratory rate on admission breath $\cdot \min ^{-1}$ & $21.2 \pm 12.0$ & $21.2 \pm 13.7$ & $21.3 \pm 4.7$ \\
\hline Heart rate on admission beat $\cdot \min ^{-1}$ & $88.7 \pm 14.6$ & $88.5 \pm 14.7$ & $89.2 \pm 14.4$ \\
\hline Systolic pressure on admission $\mathrm{mmHg}$ & $126.1 \pm 16.4$ & $123.5 \pm 15.2$ & $133.2 \pm 17.5$ \\
\hline Diastolic pressure on admission $\mathrm{mmHg}$ & $79.5 \pm 25.6$ & $79 \pm 28.9$ & $80.9 \pm 12.6$ \\
\hline Highest temperature ${ }^{\circ} \mathrm{C}$ & $38.3 \pm 1.6$ & $38.3 \pm 1.1$ & $38.2 \pm 2.6$ \\
\hline \multicolumn{4}{|l|}{ Sex } \\
\hline Male & $904 / 1578(57.3)$ & $667 / 1182(56.4)$ & $237 / 396(59.8)$ \\
\hline Female & $674 / 1578(42.7)$ & $515 / 1182(43.6)$ & $159 / 396(40.2)$ \\
\hline \multicolumn{4}{|l|}{ Smoking status } \\
\hline Never/unknown & 1479/1590 (93) & $1127 / 1191$ (94.6) & $352 / 399$ (88.2) \\
\hline Former/current & $111 / 1590(7)$ & $64 / 1191(5.4)$ & $47 / 399$ (11.8) \\
\hline \multicolumn{4}{|l|}{ Symptoms } \\
\hline Fever & $1351 / 1536(88)$ & $1002 / 1148$ (87.3) & $349 / 388$ (89.9) \\
\hline Conjunctival congestion & $10 / 1345(0.7)$ & $7 / 1014(0.7)$ & $3 / 331(0.9)$ \\
\hline Nasal congestion & $73 / 1299(5.6)$ & $59 / 979(6)$ & $14 / 320(4.4)$ \\
\hline Headache & $205 / 1328(15.4)$ & $151 / 1002(15.1)$ & $54 / 326(16.6)$ \\
\hline Dry cough & $1052 / 1498(70.2)$ & $775 / 1116(69.4)$ & $277 / 382(72.5)$ \\
\hline Pharyngodynia & $194 / 1317$ (14.7) & $148 / 999(14.8)$ & $46 / 318(14.5)$ \\
\hline Productive cough & $513 / 1424(36)$ & $363 / 1064(34.1)$ & $150 / 360(41.7)$ \\
\hline Fatigue & $584 / 1365(42.8)$ & $435 / 1031(42.2)$ & $149 / 334(44.6)$ \\
\hline Haemoptysis & $16 / 1315(1.2)$ & 9/991 (0.9) & $7 / 324(2.2)$ \\
\hline Shortness of breath & $331 / 1394(23.7)$ & $185 / 1041(17.8)$ & $146 / 353(41.4)$ \\
\hline Nausea/vomiting & $80 / 1371(5.8)$ & $44 / 1025(4.3)$ & $36 / 346(10.4)$ \\
\hline Diarrhoea & $57 / 1359(4.2)$ & $39 / 1023$ (3.8) & $18 / 336(5.4)$ \\
\hline Myalgia/arthralgia & 234/1338 (17.5) & 174/1007 (17.3) & $60 / 331(18.1)$ \\
\hline Chill & $163 / 1333(12.2)$ & $129 / 1006(12.8)$ & $34 / 327(10.4)$ \\
\hline \multicolumn{4}{|l|}{ Signs } \\
\hline Throat congestion & $21 / 1286(1.6)$ & $16 / 973(1.6)$ & $5 / 313(1.6)$ \\
\hline Tonsil swelling & $31 / 1376(2.3)$ & $22 / 1024(2.1)$ & $9 / 352(2.6)$ \\
\hline Enlargement of lymph nodes & $2 / 1375(0.1)$ & $1 / 1027(0.1)$ & $1 / 348(0.3)$ \\
\hline Rash & $3 / 1378(0.2)$ & $2 / 1032(0.2)$ & $1 / 346(0.3)$ \\
\hline Unconsciousness & $20 / 1421(1.4)$ & $11 / 1063(1)$ & $9 / 358(2.5)$ \\
\hline \multicolumn{4}{|l|}{ Abnormal chest image } \\
\hline Radiograph & 243/1590 (15.3) & $236 / 1566(15.1)$ & $44036(29.2)$ \\
\hline Computed tomography & $1130 / 1590(71.1)$ & $1113 / 1566(71.1)$ & $17 / 24(70.8)$ \\
\hline \multicolumn{4}{|l|}{ Hubei } \\
\hline Yes & $647 / 1590(40.7)$ & 434/1191 (36.4) & $213 / 399(53.4)$ \\
\hline No & $943 / 1590(59.3)$ & $757 / 1191(63.6)$ & $186 / 399(46.6)$ \\
\hline \multicolumn{4}{|l|}{ Wuhan contacted } \\
\hline Yes & 1334/1590 (83.9) & $983 / 1191(82.5)$ & $351 / 399(88)$ \\
\hline No & $256 / 1590(16.1)$ & $208 / 1191(17.5)$ & $48 / 399(12)$ \\
\hline Severity & $254 / 1590(16)$ & $123 / 1191(10.3)$ & $131 / 399$ (32.8) \\
\hline Composite end-point & $131 / 1590(8.2)$ & $54 / 1191(4.5)$ & $77 / 399$ (19.3) \\
\hline Death & $50 / 1590(3.1)$ & $15 / 1191(1.3)$ & $35 / 399(8.8)$ \\
\hline Admission to ICU & $99 / 1590(6.2)$ & 45/1191 (3.8) & $54 / 399$ (13.5) \\
\hline Invasive ventilation & $50 / 1590(3.1)$ & $19 / 1191(1.6)$ & $31 / 399(1.6)$ \\
\hline
\end{tabular}

Data are presented as mean $\pm \mathrm{SD}$ or $\mathrm{n} / \mathrm{N}(\%)$, where $\mathrm{N}$ is the total number of patients with available data. ICU: intensive care unit.

\section{Discussion}

Our study is the first nationwide investigation that systematically evaluates the impact of comorbidities on the clinical characteristics and prognosis in patients with COVID-19 in China. Circulatory and endocrine comorbidities were common among patients with COVID-19. Patients with at least one comorbidity, or 
TABLE 2 Demographics and clinical characteristics of patients with 1 or $\geqslant 2$ comorbidities

\begin{tabular}{|c|c|c|}
\hline Variables & 1 comorbidity & $\geqslant 2$ comorbidities \\
\hline Subjects $n$ & 269 & 130 \\
\hline Age years & $58.2 \pm 13.1$ & $66.2 \pm 12.2$ \\
\hline Incubation period days & $3.2 \pm 3.1$ & $4.0 \pm 5.2$ \\
\hline Temperature on admission ${ }^{\circ} \mathrm{C}$ & $37.4 \pm 0.9$ & $37.1 \pm 0.9$ \\
\hline Respiratory rate on admission breath $\cdot \min ^{-1}$ & $21.4 \pm 4.6$ & $21.2 \pm 5$ \\
\hline Heart rate beat $\cdot \min ^{-1}$ & $90.2 \pm 14.6$ & $87.2 \pm 13.7$ \\
\hline Systolic pressure on admission $\mathrm{mmHg}$ & $132.2 \pm 16.5$ & $135.3 \pm 19.4$ \\
\hline Diastolic pressure on admission $\mathrm{mmHg}$ & $81.7 \pm 12.5$ & $79.5 \pm 12.9$ \\
\hline Highest temperature ${ }^{\circ} \mathrm{C}$ & $38.2 \pm 3.0$ & $38.4 \pm 0.8$ \\
\hline \multicolumn{3}{|l|}{ Sex } \\
\hline Male & $158 / 268(59.0)$ & $79 / 128$ (61.7) \\
\hline Female & $110 / 268(41.0)$ & $49 / 128$ (38.3) \\
\hline \multicolumn{3}{|l|}{ Smoking status } \\
\hline Never/unknown & $234 / 269(87.0)$ & $118 / 130(90.8)$ \\
\hline Former/current & $35 / 269(13.0)$ & $12 / 130(9.2)$ \\
\hline \multicolumn{3}{|l|}{ Symptoms } \\
\hline Fever & $241 / 263(91.6)$ & $108 / 125(86.4)$ \\
\hline Conjunctival congestion & $3 / 222(1.4)$ & $0 / 109(0)$ \\
\hline Nasal congestion & $5 / 213(2.3)$ & $9 / 107(8.4)$ \\
\hline Headache & $34 / 220$ (15.5) & $20 / 106$ (18.9) \\
\hline Dry cough & $195 / 258(75.6)$ & $82 / 124(66.1)$ \\
\hline Pharyngodynia & $33 / 218(15.1)$ & $13 / 100(13.0)$ \\
\hline Productive cough & $101 / 241(41.9)$ & $49 / 119(41.2)$ \\
\hline Fatigue & $97 / 227(42.7)$ & $52 / 107(48.6)$ \\
\hline Haemoptysis & $4 / 219(1.8)$ & $3 / 105$ (2.9) \\
\hline Shortness of breath & $79 / 232(34.1)$ & $67 / 121(55.4)$ \\
\hline Nausea/vomiting & $23 / 236(9.7)$ & $13 / 110$ (11.8) \\
\hline Diarrhoea & $11 / 229(4.8)$ & $7 / 107(6.5)$ \\
\hline Myalgia/arthralgia & $45 / 227(19.8)$ & $15 / 104(14.4)$ \\
\hline Chill & $25 / 222(11.3)$ & $9 / 105(8.6)$ \\
\hline \multicolumn{3}{|l|}{ Signs } \\
\hline Throat congestion & $4 / 216(1.9)$ & $1 / 97(1)$ \\
\hline Tonsil swelling & $5 / 234(2.1)$ & $4 / 118(3.4)$ \\
\hline Enlargement of lymph nodes & $1 / 232(0.4)$ & $0 / 116(0)$ \\
\hline Rash & $0 / 231(0)$ & $1 / 115(0.9)$ \\
\hline Unconsciousness & $3 / 240(1.3)$ & $6 / 118(5.1)$ \\
\hline \multicolumn{3}{|l|}{ Abnormal chest image } \\
\hline Radiograph & $63 / 269(23.4)$ & $27 / 130$ (20.8) \\
\hline Computed tomography & $200 / 269(74.3)$ & $96 / 130(73.8)$ \\
\hline \multicolumn{3}{|l|}{ Hubei } \\
\hline Yes & $120 / 269(44.6)$ & $93 / 130$ (71.5) \\
\hline No & $149 / 269(55.4)$ & $37 / 130$ (28.5) \\
\hline \multicolumn{3}{|l|}{ Wuhan contacted } \\
\hline Yes & $229 / 269(85.1)$ & $122 / 130$ (93.8) \\
\hline No & $40 / 269(14.9)$ & $8 / 130(6.2)$ \\
\hline Severity & $79 / 269$ (29.4) & $52 / 130(40.0)$ \\
\hline Composite end-point & $40 / 269(14.9)$ & $37 / 130$ (28.5) \\
\hline Deaths & $15 / 269(5.6)$ & $20 / 130(15.4)$ \\
\hline Admission to ICU & $31 / 269$ (11.5) & $23 / 130$ (17.7) \\
\hline Invasive ventilation & $17 / 269(6.3)$ & $14 / 130$ (10.8) \\
\hline
\end{tabular}

even more so, were associated with poor clinical outcomes. These findings have provided further objective evidence, with a large sample size and extensive coverage of the geographic regions across China, to take into account baseline comorbid diseases in the comprehensive risk assessment of prognosis among patients with COVID-19 on hospital admission.

Overall, our findings have echoed the recently published studies in terms of the commonness of comorbidities in patients with COVID-19 [3-7]. Despite considerable variations in the proportion in 
TABLE 3 Demographics and clinical characteristics of patients stratified by different comorbidities

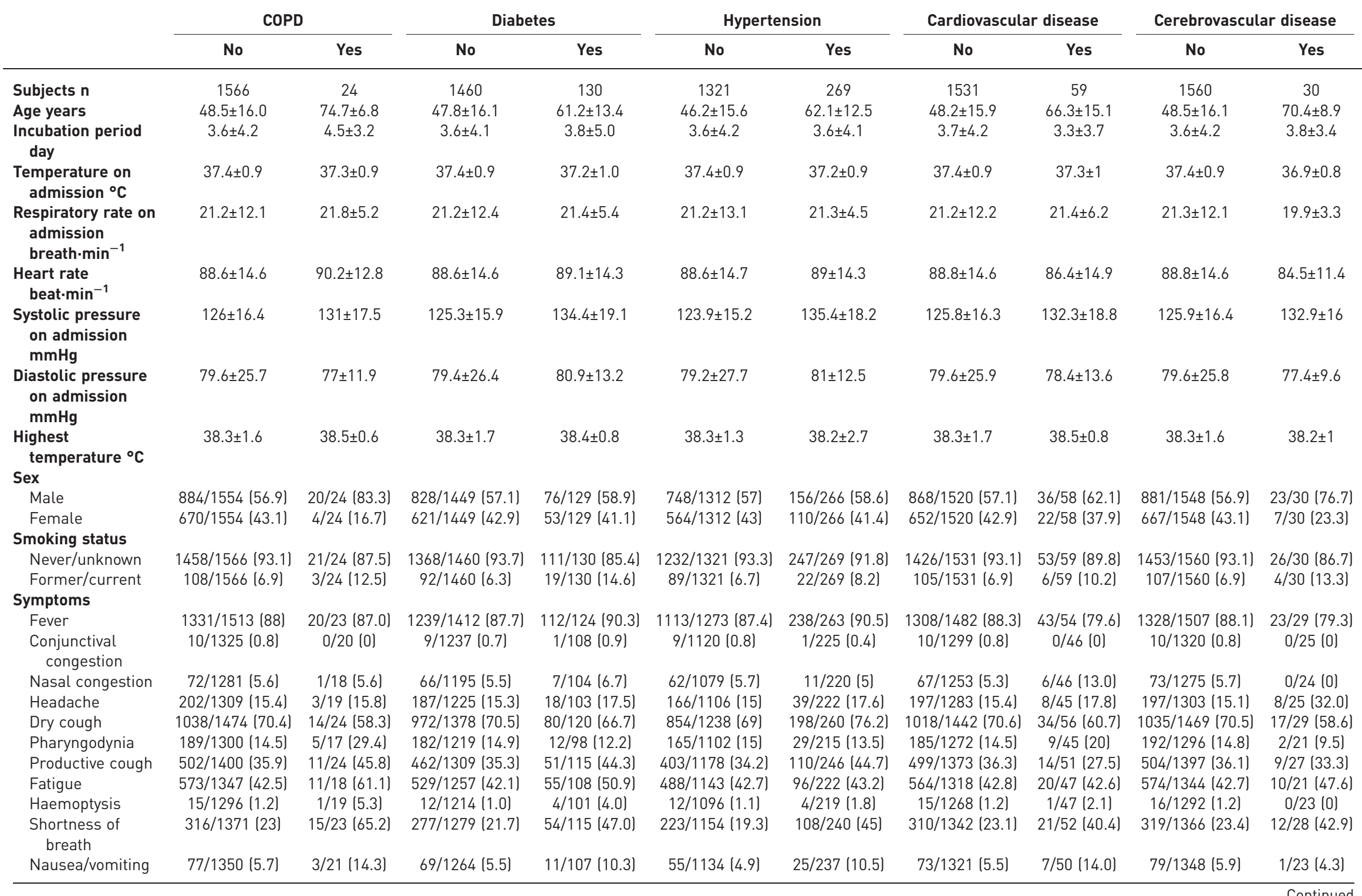




\begin{tabular}{|c|c|c|c|c|c|c|c|c|c|c|}
\hline & \multicolumn{2}{|c|}{ COPD } & \multicolumn{2}{|c|}{ Diabetes } & \multicolumn{2}{|c|}{ Hypertension } & \multicolumn{2}{|c|}{ Cardiovascular disease } & \multicolumn{2}{|c|}{ Cerebrovascular disease } \\
\hline & No & Yes & No & Yes & No & Yes & No & Yes & No & Yes \\
\hline Diarrhoea & $57 / 1338$ (4.3) & $0 / 21(0)$ & 48/1255 (3.8) & $9 / 104(8.7)$ & $46 / 1129(4.1)$ & $11 / 230(4.8)$ & $53 / 1313(4.0)$ & $4 / 46(8.7)$ & $57 / 1336(4.3)$ & $0 / 23(0)$ \\
\hline $\begin{array}{l}\text { Myalgia/ } \\
\text { arthralgia }\end{array}$ & $231 / 1320(17.5)$ & $3 / 18(16.7)$ & 218/1234 (17.7) & $16 / 104(15.4)$ & 188/1112 (16.9) & $46 / 226(20.4)$ & $227 / 1294$ (17.5) & $7 / 44(15.9)$ & 233/1317 (17.7) & $1 / 21(4.8)$ \\
\hline Chill & $159 / 1313(12.1)$ & $4 / 20(20.0)$ & $151 / 1230(12.3)$ & $12 / 103(11.7)$ & $140 / 1111(12.6)$ & $23 / 222(10.4)$ & $161 / 1290(12.5)$ & $2 / 43(4.7)$ & $162 / 1310(12.4)$ & $1 / 23(4.3)$ \\
\hline \multicolumn{11}{|l|}{ Signs } \\
\hline $\begin{array}{l}\text { Throat } \\
\text { congestion }\end{array}$ & $21 / 1269(1.7)$ & $0 / 17(0)$ & $20 / 1189(1.7)$ & $1 / 97(1)$ & 18/1075 (1.7) & $3 / 211(1.4)$ & $21 / 1245(1.7)$ & $0 / 41(0)$ & $21 / 1266(1.7)$ & $0 / 20(0)$ \\
\hline Tonsil swelling & $31 / 1355$ (2.3) & $0 / 21(0)$ & $28 / 1265(2.2)$ & $3 / 111(2.7)$ & $25 / 1133(2.2)$ & $6 / 243(2.5)$ & $29 / 1326(2.2)$ & $2 / 50(4.0)$ & $31 / 1348(2.3)$ & $0 / 28(0)$ \\
\hline $\begin{array}{c}\text { Enlargement of } \\
\text { lymph nodes }\end{array}$ & $2 / 1355(0.1)$ & $0 / 20(0)$ & $2 / 1267(0.2)$ & $0 / 108(0)$ & $2 / 1135(0.2)$ & $0 / 240(0)$ & $1 / 1325(0.1)$ & $1 / 50(2.0)$ & $2 / 1347(0.1)$ & $0 / 28(0)$ \\
\hline Rash & $3 / 1357(0.2)$ & $0 / 21(0)$ & $2 / 1270(0.2)$ & $1 / 108(0.9)$ & $2 / 1141(0.2)$ & $1 / 237(0.4)$ & $3 / 1327(0.2)$ & $0 / 51(0)$ & $3 / 1351(0.2)$ & $0 / 27(0)$ \\
\hline Unconsciousness & $18 / 1400$ (1.3) & $2 / 21(9.5)$ & $18 / 1309$ (1.4) & $2 / 112(1.8)$ & $12 / 1175$ (1.0) & $8 / 246$ (3.3) & $17 / 1371(1.2)$ & $3 / 50(6)$ & $19 / 1392(1.4)$ & $1 / 29(3.4)$ \\
\hline \multicolumn{11}{|l|}{$\begin{array}{l}\text { Abnormal chest } \\
\text { image }\end{array}$} \\
\hline Radiograph & $236 / 1566(15.1)$ & $7 / 24(29.2)$ & $218 / 1460(14.9)$ & $25 / 130(19.2)$ & $178 / 1321(13.5)$ & $65 / 269(24.2)$ & $231 / 1531(15.1)$ & $12 / 59(20.3)$ & $231 / 1560$ (14.8) & $12 / 30(40)$ \\
\hline $\begin{array}{l}\text { Computed } \\
\text { tomography }\end{array}$ & $1113 / 1566(71.1)$ & $17 / 24(70.8)$ & $1034 / 1460(70.8)$ & $96 / 130(73.8)$ & $926 / 1321(70.1)$ & $204 / 269(75.8)$ & $1090 / 1531$ (71.2) & $40 / 59(67.8)$ & $1111 / 1560$ (71.2) & $19 / 30$ (63.3) \\
\hline \multicolumn{11}{|l|}{ Hubei } \\
\hline Yes & $633 / 1566$ (40.4) & $14 / 24$ (58.3) & $568 / 1460$ (38.9) & $79 / 130$ (60.8) & 491/1321 (37.2) & $156 / 269(58)$ & 609/1531 (39.8) & $38 / 59(64.4)$ & $623 / 1560$ (39.9) & $24 / 30(80.0)$ \\
\hline No & $933 / 1566$ (59.6) & $10 / 24(41.7)$ & $892 / 1460(61.1)$ & $51 / 130(39.2)$ & $830 / 1321$ (62.8) & $113 / 269$ (42) & $922 / 1531(60.2)$ & $21 / 59(35.6)$ & $937 / 1560(60.1)$ & $6 / 30(20.0)$ \\
\hline \multicolumn{11}{|l|}{ Wuhan contacted } \\
\hline Yes & $1312 / 1566$ (83.8) & $22 / 24$ (91.7) & $1216 / 1460$ (83.3) & $118 / 130$ (90.8) & $1092 / 1321$ (82.7) & $242 / 269(90)$ & $1282 / 1531$ (83.7) & $52 / 59(88.1)$ & $1306 / 1560$ (83.7) & $28 / 30$ (93.3) \\
\hline No & $254 / 1566$ (16.2) & $2 / 24(8.3)$ & $244 / 1460(16.7)$ & $12 / 130(9.2)$ & $229 / 1321$ (17.3) & $27 / 269(10)$ & $249 / 1531$ (16.3) & $7 / 59(11.9)$ & $254 / 1560(16.3)$ & $2 / 30(6.7)$ \\
\hline Severity & $239 / 1566$ (15.3) & $15 / 24(62.5)$ & $209 / 1460$ (14.3) & $45 / 130(34.6)$ & $166 / 1321(12.6)$ & $88 / 269$ (32.7) & 234/1531 (15.3) & $20 / 59$ (33.9) & $239 / 1560$ (15.3) & $15 / 30(50)$ \\
\hline $\begin{array}{l}\text { Composite } \\
\text { end-point }\end{array}$ & $119 / 1566(7.6)$ & $12 / 24(50.0)$ & $100 / 1460(6.8)$ & $31 / 130$ (23.8) & $78 / 1321$ (5.9) & $53 / 269$ (19.7) & $118 / 1531$ (7.7) & $13 / 59(22.0)$ & $121 / 1560(7.8)$ & $10 / 30$ (33.3) \\
\hline Deaths & $44 / 1566(2.8)$ & $6 / 24(25.0)$ & $37 / 1460(2.5)$ & $13 / 130(10.0)$ & $22 / 1321$ (1.7) & $28 / 269(10.4)$ & 42/1531 (2.7) & $8 / 59(13.6)$ & $44 / 1560(2.8)$ & $6 / 30(20)$ \\
\hline Admission to ICU & $92 / 1566$ (5.9) & $7 / 24(29.2)$ & $80 / 1460(5.5)$ & $19 / 130(14.6)$ & $61 / 1321(4.6)$ & $38 / 269(14.1)$ & $91 / 1531$ (5.9) & $8 / 59(13.6)$ & $92 / 1560$ (5.9) & $7 / 30(23.3)$ \\
\hline Invasive ventilation & $45 / 1566$ (2.9) & $5 / 24(20.8)$ & $39 / 1460(2.7)$ & $11 / 130(8.5)$ & $28 / 1321(2.1)$ & $22 / 269(8.2)$ & $44 / 1531$ (2.9) & $6 / 59(10.2)$ & $46 / 1560(2.9)$ & $4 / 30$ (13.3) \\
\hline
\end{tabular}




\begin{tabular}{|c|c|c|c|c|c|c|c|c|}
\hline & \multicolumn{2}{|c|}{ Hepatitis B infection } & \multicolumn{2}{|c|}{ Malignancy } & \multicolumn{2}{|c|}{ Chronic kidney disease } & \multicolumn{2}{|c|}{ Immunodeficiency } \\
\hline & No & Yes & No & Yes & No & Yes & No & Yes \\
\hline Subjects $\mathrm{n}$ & 1562 & 28 & 1572 & 18 & 1569 & 21 & 1587 & 3 \\
\hline Age years & $48.9 \pm 16.3$ & $50.8 \pm 14.8$ & $48.7 \pm 16.2$ & $63.1 \pm 12.1$ & $48.8 \pm 16.2$ & $63.7 \pm 14$ & $48.9 \pm 16.3$ & $51 \pm 21.7$ \\
\hline Incubation period day & $3.7 \pm 4.2$ & $3 \pm 2.8$ & $3.7 \pm 4.2$ & $3.1 \pm 3.1$ & $3.6 \pm 4.1$ & $3.3 \pm 7.5$ & $3.6 \pm 4.1$ & $12.7 \pm 16.3$ \\
\hline Temperature on admission ${ }^{\circ} \mathrm{C}$ & $37.4 \pm 0.9$ & $37.3 \pm 0.8$ & $37.4 \pm 0.9$ & $37.3 \pm 0.9$ & $37.4 \pm 0.9$ & $37.2 \pm 1$ & $37.4 \pm 0.9$ & $36.6 \pm 0.2$ \\
\hline Respiratory rate on admission breath $\cdot \min ^{-1}$ & $21.2 \pm 12.1$ & $21.2 \pm 3$ & $21.3 \pm 12.1$ & $20.2 \pm 1.6$ & $21.3 \pm 12.1$ & $19 \pm 2.8$ & $21.3 \pm 12$ & $19 \pm 1$ \\
\hline Heart rate beat $\cdot \min ^{-1}$ & $88.7 \pm 14.6$ & $86.3 \pm 13.2$ & $88.7 \pm 14.6$ & $89.4 \pm 13.1$ & $88.7 \pm 14.6$ & $89.1 \pm 12.5$ & $88.7 \pm 14.6$ & $91 \pm 18.5$ \\
\hline Systolic pressure on admission $\mathrm{mmHg}$ & $126.1 \pm 16.4$ & $124.8 \pm 14.7$ & $126 \pm 16.4$ & $128.3 \pm 14.5$ & $125.9 \pm 16.3$ & $135.4 \pm 20.5$ & $126.1 \pm 16.4$ & $127.3 \pm 7.4$ \\
\hline Diastolic pressure on admission $\mathrm{mmHg}$ & $79.6 \pm 25.7$ & $78.3 \pm 13$ & $79.5 \pm 25.7$ & $81.2 \pm 8.8$ & $79.5 \pm 25.7$ & $79.8 \pm 14$ & $79.5 \pm 25.6$ & $84.7 \pm 15$ \\
\hline Highest temperature ${ }^{\circ} \mathrm{C}$ & $38.3 \pm 1.5$ & $37.6 \pm 4.4$ & $38.3 \pm 1.6$ & $38.5 \pm 0.9$ & $38.3 \pm 1.6$ & $38.5 \pm 0.5$ & $38.3 \pm 1.6$ & $38 \pm 0.5$ \\
\hline \multicolumn{9}{|l|}{ Sex } \\
\hline Male & $885 / 1550(57.1)$ & $19 / 28(67.9)$ & $893 / 1560(57.2)$ & $11 / 18(61.1)$ & $891 / 1559(57.2)$ & $13 / 19(68.4)$ & $904 / 1575(57.4)$ & $0 / 3(0)$ \\
\hline Female & $665 / 1550$ (42.9) & $9 / 28(32.1)$ & $667 / 1560(42.8)$ & $7 / 18(38.9)$ & $668 / 1559(42.8)$ & $6 / 19(31.6)$ & 671/1575 (42.6) & $3 / 3(100)$ \\
\hline \multicolumn{9}{|l|}{ Smoking status } \\
\hline Never/unknown & 1454/1562 (93.1) & 25/28 (89.3) & 1465/1572 (93.2) & 14/18 (77.8) & 1459/1569 (93) & $20 / 21(95.2)$ & 1477/1587 (93.1) & $2 / 3(66.7)$ \\
\hline Former/current & 108/1562 (6.9) & $3 / 28$ (10.7) & 107/1572 (6.8) & 4/18 (22.2) & 110/1569 (7) & $1 / 21(4.8)$ & 110/1587 (6.9) & $1 / 3(33.3)$ \\
\hline \multicolumn{9}{|l|}{ Symptoms } \\
\hline Fever & 1326/1508 (87.9) & 25/28 (89.3) & 1335/1519 (87.9) & $16 / 17(94.1)$ & $1334 / 1516(88)$ & $17 / 20(85)$ & 1348/1533 (87.9) & $3 / 3(100)$ \\
\hline $\begin{array}{l}\text { Conjunctival } \\
\text { congestion }\end{array}$ & $9 / 1323(0.7)$ & $1 / 22$ (4.5) & $10 / 1330(0.8)$ & $0 / 15(0)$ & $10 / 1328(0.8)$ & $0 / 17(0)$ & $10 / 1343(0.7)$ & $0 / 2(0)$ \\
\hline Nasal congestion & $73 / 1277$ (5.7) & $0 / 22(0)$ & $71 / 1285$ (5.5) & $2 / 14(14.3)$ & $73 / 1282$ (5.7) & 0/17 (0) & $73 / 1297$ (5.6) & $0 / 2(0)$ \\
\hline Headache & $202 / 1306$ (15.5) & $3 / 22(13.6)$ & $203 / 1314(15.4)$ & $2 / 14(14.3)$ & $203 / 1311$ (15.5) & 2/17 (11.8) & $205 / 1326$ (15.5) & $0 / 2(0)$ \\
\hline Dry cough & $1037 / 1472(70.4)$ & $15 / 26(57.7)$ & 1039/1481 (70.2) & 13/17 (76.5) & $1037 / 1479(70.1)$ & $15 / 19(78.9)$ & $1050 / 1495(70.2)$ & $2 / 3(66.7)$ \\
\hline Pharyngodynia & 188/1294 (14.5) & $6 / 23(26.1)$ & 193/1303 (14.8) & $1 / 14(7.1)$ & $191 / 1300(14.7)$ & $3 / 17(17.6)$ & 193/1315 (14.7) & $1 / 2(50)$ \\
\hline Productive cough & $508 / 1401$ (36.3) & $5 / 23(21.7)$ & $504 / 1408$ (35.8) & $9 / 16(56.3)$ & $505 / 1407$ (35.9) & $8 / 17(47.1)$ & $512 / 1421(36)$ & $1 / 3$ (33.3) \\
\hline Fatigue & $570 / 1340$ (42.5) & $14 / 25(56)$ & $577 / 1349$ (42.8) & $7 / 16$ (43.8) & $581 / 1350(43)$ & $3 / 15(20)$ & $583 / 1363$ (42.8) & $1 / 2(50)$ \\
\hline Haemoptysis & $16 / 1293(1.2)$ & $0 / 22(0)$ & $15 / 1299(1.2)$ & $1 / 16$ (6.3) & $16 / 1300(1.2)$ & $0 / 15(0)$ & $16 / 1313$ (1.2) & $0 / 2(0)$ \\
\hline $\begin{array}{l}\text { Shortness of } \\
\text { breath }\end{array}$ & $321 / 1370(23.4)$ & 10/24 (41.7) & $323 / 1377$ (23.5) & $8 / 17(47.1)$ & $321 / 1375$ (23.3) & $10 / 19(52.6)$ & $330 / 1392(23.7)$ & $1 / 2(50)$ \\
\hline Nausea/vomiting & $78 / 1349$ (5.8) & $2 / 22(9.1)$ & 78/1355 (5.8) & $2 / 16$ (12.5) & $79 / 1351$ (5.8) & $1 / 20(5)$ & $80 / 1369$ (5.8) & $0 / 2(0)$ \\
\hline Diarrhoea & $55 / 1337(4.1)$ & $2 / 22(9.1)$ & $57 / 1343$ (4.2) & $0 / 16(0)$ & $56 / 1339(4.2)$ & $1 / 20(5)$ & $56 / 1356(4.1)$ & $1 / 3$ (33.3) \\
\hline Myalgia/arthralgia & $232 / 1316(17.6)$ & $2 / 22(9.1)$ & $231 / 1322(17.5)$ & $3 / 16(18.8)$ & $233 / 1323(17.6)$ & $1 / 15(6.7)$ & $233 / 1336(17.4)$ & $1 / 2(50)$ \\
\hline Chill & $161 / 1310(12.3)$ & 2/23 (8.7) & $162 / 1318$ (12.3) & $1 / 15$ (6.7) & $161 / 1317(12.2)$ & $2 / 16$ (12.5) & $163 / 1331(12.2)$ & $0 / 2(0)$ \\
\hline \multicolumn{9}{|l|}{ Signs } \\
\hline Throat congestion & $21 / 1264(1.7)$ & $0 / 22(0)$ & 20/1271 (1.6) & $1 / 15$ (6.7) & 21/1271 (1.7) & 0/15 (0) & 20/1284 (1.6) & $1 / 2(50)$ \\
\hline Tonsil swelling & $30 / 1353(2.2)$ & $1 / 23$ (4.3) & $30 / 1359(2.2)$ & $1 / 17(5.9)$ & $30 / 1356(2.2)$ & $1 / 20(5)$ & $31 / 1373$ (2.3) & $0 / 3(0)$ \\
\hline $\begin{array}{c}\text { Enlargement of } \\
\text { lymph nodes }\end{array}$ & $2 / 1352(0.1)$ & $0 / 23(0)$ & $2 / 1359(0.1)$ & $0 / 16(0)$ & $2 / 1355(0.1)$ & $0 / 20(0)$ & $2 / 1372(0.1)$ & $0 / 3(0)$ \\
\hline Rash & $3 / 1355(0.2)$ & $0 / 23(0)$ & $3 / 1361(0.2)$ & $0 / 17(0)$ & $3 / 1360(0.2)$ & $0 / 18(0)$ & $3 / 1376$ (0.2) & $0 / 2(0)$ \\
\hline Unconsciousness & $19 / 1397(1.4)$ & $1 / 24(4.2)$ & $20 / 1404$ (1.4) & $0 / 17(0)$ & $20 / 1401$ (1.4) & $0 / 20(0)$ & $20 / 1418$ (1.4) & $0 / 3(0)$ \\
\hline \multicolumn{9}{|l|}{ Abnormal chest image } \\
\hline Radiograph & $240 / 1562(15.4)$ & $3 / 28$ (10.7) & 239/1572 (15.2) & 4/18 (22.2) & $240 / 1569$ (15.3) & $3 / 21(14.3)$ & 243/1587 (15.3) & $0 / 3(0)$ \\
\hline $\begin{array}{l}\text { Computed } \\
\text { tomography }\end{array}$ & $1111 / 1562(71.1)$ & 19/28 (67.9) & $1113 / 1572$ (70.8) & $17 / 18(94.4)$ & $1116 / 1569(71.1)$ & $14 / 21(66.7)$ & 1127/1587 (71) & $3 / 3(100)$ \\
\hline
\end{tabular}




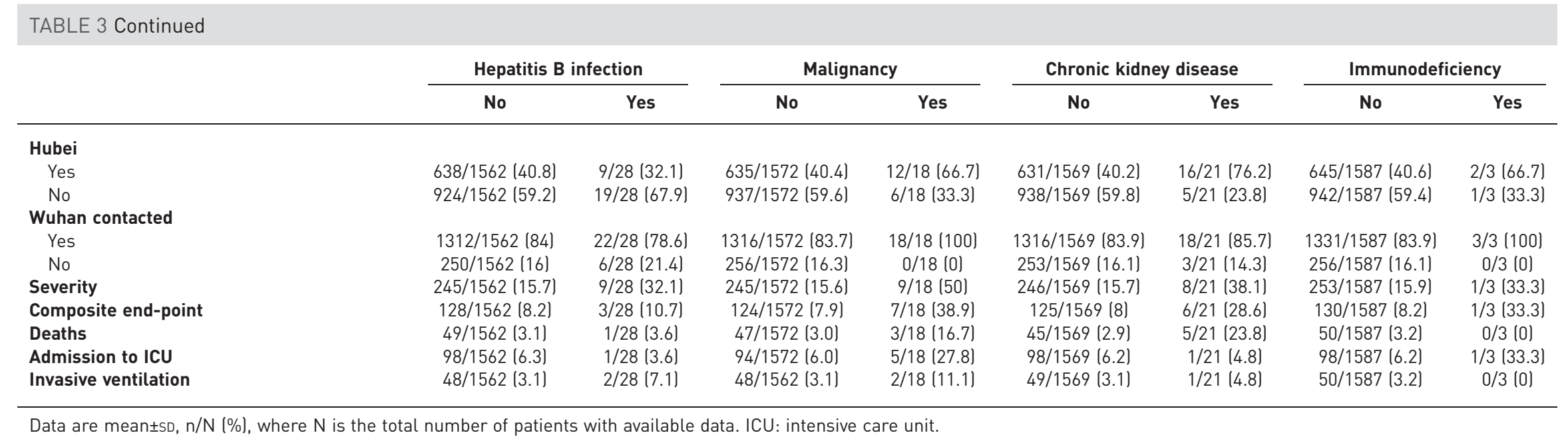



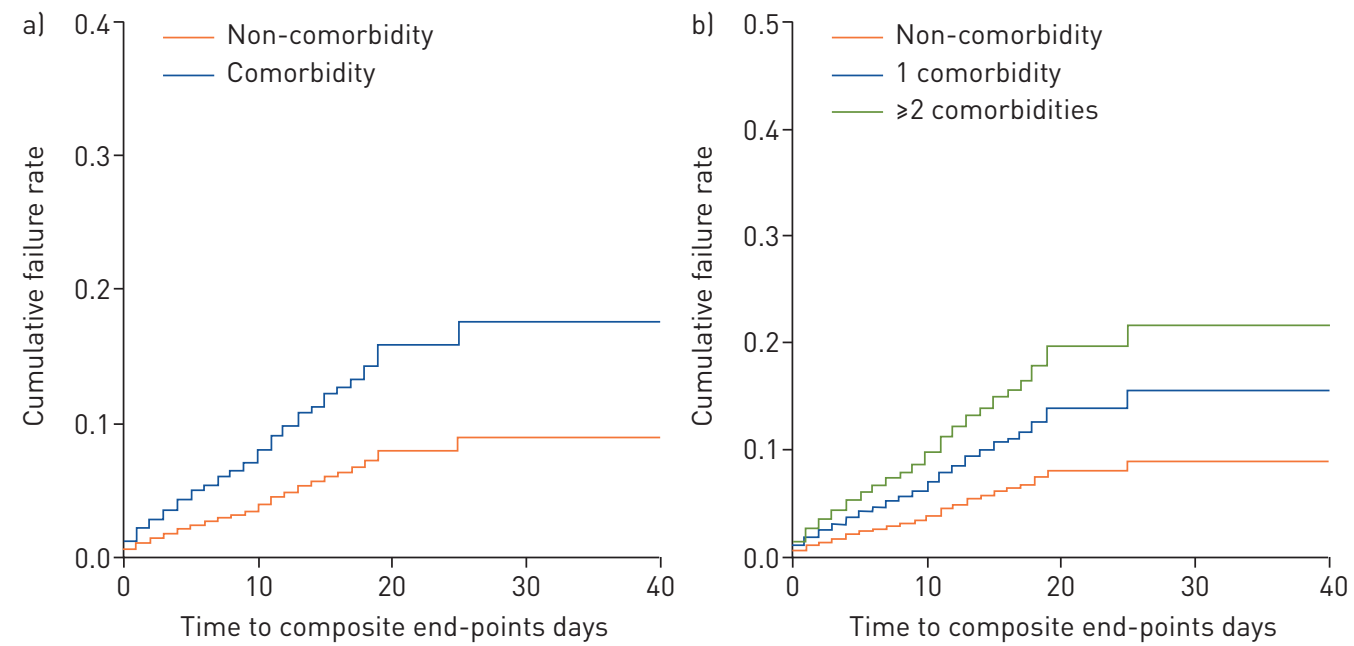

FIGURE 1 a) The time-dependent risk of reaching the composite end-points between patients with or without any comorbidity. b) The time-dependent risk of reaching the composite end-points between patients without any comorbidity, patients with a single comorbidity and patients with two or more comorbidities. Cox proportional hazard regression models were applied to determine the potential risk factors associated with the composite end-points, with the hazard ratio and $95 \%$ confidence interval being reported.

individual studies due to the limited sample size and the region where patients were managed, circulatory diseases (including hypertension and coronary heart diseases) remained the most common category of comorbidity [3-7]. Apart from circulatory diseases, endocrine diseases such as diabetes were also common in patients with COVID-19. Notwithstanding the commonness of circulatory and endocrine comorbidities, patients with COVID-19 rarely reported having comorbid respiratory diseases (particularly COPD). The reasons underlying this observation have been scant, but could have arisen from the lack of awareness and the lack of spirometric testing in community settings that collectively contributed to the under-diagnosis of respiratory diseases [33]. It should be stressed that the observed frequency of comorbidity may also reflect the transmission dynamics within particular age groups, case detection or testing practices or hospital admission policies during the early phases of the epidemic. Consistent with recent reports [3-7], the percentage of patients with comorbid renal disease and malignancy was relatively low. Our findings have therefore added to the existing literature on the spectrum of comorbidities in patients with COVID-19 based on the larger sample sizes and representativeness of the whole patient population in China.

A number of existing literature reports have documented the escalated risks of poorer clinical outcomes in patients with avian influenza [14-18], SARS-CoV [19] and MERS-CoV infections [20-28]. The most common comorbidities associated with poorer prognosis included diabetes [25, 29], hypertension [28],

\begin{tabular}{|c|c|c|c|}
\hline \multicolumn{2}{|l|}{ Features } & \multirow[t]{2}{*}{$\operatorname{HR}(95 \% \mathrm{CI})$} & \multirow[t]{2}{*}{$p$-value } \\
\hline Type of comorbidities & & & \\
\hline COPD & $\mapsto$ & 2.681 (1.424-5.058) & 0.002 \\
\hline Diabetes & $-1-1$ & $1.586(1.028-2.449)$ & 0.037 \\
\hline Hypertension & -4 & 1.575 (1.069-2.322) & 0.022 \\
\hline Malignant tumour & $\longmapsto$ & $3.501(1.604-7.643)$ & 0.002 \\
\hline \multicolumn{4}{|l|}{ Comorbidities $\mathrm{n}$} \\
\hline 1 & $H=-1$ & $1.789(1.155-2.772)$ & 0.009 \\
\hline$\geqslant 2$ & $1-1$ & $2.592(1.611-4.171)$ & $<0.001$ \\
\hline
\end{tabular}

FIGURE 2 Predictors of the composite end-points in the proportional hazards model. Hazard ratio $195 \%$ confidence interval) are shown for the risk factors associated with the composite end-points (admission to intensive care unit, invasive ventilation or death). The comorbidities were classified according to the organ systems as well as the number. The scale bar indicates the hazard ratio. Cox proportional hazard regression models were applied to determine the potential risk factors associated with the composite end-points, with the hazard ratio $195 \%$ confidence interval) being reported. The model has been adjusted with age and smoking status. 
respiratory diseases $[19,28]$, cardiac diseases [19, 28], pregnancy [16], renal diseases [28] and malignancy [19]. Our findings suggested that, similar with other severe acute respiratory outbreaks, comorbidities such as COPD, diabetes, hypertension and malignancy predisposed to adverse clinical outcomes in patients with COVID-19. The strength of association between different comorbidities and the prognosis, however, was less consistent when compared with the literature reports $[16,19,25,28]$. For instance, the risk between cardiac diseases and poor clinical outcomes of influenza, SARS-CoV or MERS-CoV infections was inconclusive $[16,19,25,28]$. Except for diabetes, no other comorbidities were identified to be the predictors of poor clinical outcomes in patients with MERS-CoV infections [25]. Few studies, however, have explored the mechanisms underlying these associations. KULSCAR et al. [27] showed that MERS-CoV infections resulted in prolonged airway inflammation, immune cell dysfunction and an altered expression profile of inflammatory mediators in diabetic mice models. A network-based analysis indicated that SARS-CoV infections led to immune dysregulation that could help explain the escalated risk of cardiac diseases, bone diseases and malignancy [34]. Therefore, immune dysregulation and prolonged inflammation might be the key drivers of the poor clinical outcomes in patients with COVID-19 but await verification in more mechanistic studies.

It has been well accepted that some comorbidities frequently coexist. For instance, diabetes [35] and COPD [36] frequently coexist with hypertension or coronary heart diseases. Therefore, patients with coexisting comorbidities are more likely to have poorer baseline well-being. Importantly, we have verified the significantly escalated risk of poor prognosis in patients with two or more comorbidities compared with those who had no or only a single comorbidity. Our findings implied that both the category and number of comorbidities should be taken into account when predicting the prognosis in patients with COVID-19.

Our findings suggested that patients with comorbidities had greater disease severity compared with those without. Furthermore, a greater number of comorbidities correlated with greater disease severity of COVID-19. The proper triage of patients should be implemented by carefully inquiring about the medical history because this will help identify patients who would be more likely to develop serious adverse outcomes of COVID-19. Moreover, better protection should be given to the patients with COIVD-19 who had comorbidities upon confirmation of the diagnosis.

A main limitation was the self-reporting of comorbidities on admission. Under-reporting of comorbidities, which could have stemmed from the lack of awareness and/or the lack of diagnostic testing, might contribute to the underestimation of the true strength of association with the clinical prognosis. Under-reporting of comorbidities could also lead to over-estimation of strength of association with adverse outcome. However, significant under-reporting was unlikely because the spectrum of our report was largely consistent with existing literature [3-7] and all patients were subject to a thorough history taking after hospital admission. The relatively low age might help explain the low prevalence of COPD in our cohort. Moreover, the duration of follow-up was relatively short and some patients remained in hospital at the time of writing. More studies that explore the associations in a sufficiently long time-frame are warranted. Caution should be exercised when extrapolating our findings to other countries where there are outbreaks of COVID-19 since the prevalence of comorbidities may differ among different countries. Therefore, future studies that include an external validation of the results would be desirable. Although the temperature and systolic blood pressure differed between some subgroups, they were unlikely to be clinically relevant. Finally, because of the rapid evolving outbreak globally, ongoing studies with the inclusion of more patients would be needed to increase the statistical power and lend support to subgroup analyses stratified by the specific comorbidities (i.e. COPD) and their association with the risk of death.

\section{Conclusions}

Among laboratory confirmed cases of COVID-19, patients with any comorbidity yielded poorer clinical outcomes than those without. A greater number of comorbidities also correlated with poorer clinical outcomes. A thorough assessment of comorbidities may help establish risk stratification of patients with COVID-19 upon hospital admission.

Acknowledgement: We thank the hospital staff (please refer to the appendix in the supplementary material for the full list) for their efforts in collecting the information. We are indebted to the coordination of Zong-jiu Zhang, Ya-hui Jiao, Bin Du, Xin-qiang Gao and Tao Wei (National Health Commission), Yu-fei Duan and Zhi-ling Zhao (Health Commission of Guangdong Province), Yi-min Li, Zi-jing Liang, Nuo-fu Zhang, Shi-yue Li, Qing-hui Huang, Wen-xi Huang and Ming Li (Guangzhou Institute of Respiratory Health) who greatly facilitated the collection of patient's data. Special thanks are given to the statistical team members Zheng Chen, Dong Han, Li, Zheng Chen, Zhi-ying Zhan, Jin-jian Chen, Li-jun Xu and Xiao-han Xu (State Key Laboratory of Organ Failure Research, Dept of Biostatistics, Guangdong Provincial Key Laboratory of Tropical Disease Research, School of Public Health, Southern Medical University). We also thank Li-qiang Wang, Wei-peng Cai and Zi-sheng Chen (Sixth Affiliated Hospital of Guangzhou Medical University), Chang-xing Ou, Xiao-min Peng, Si-ni Cui, Yuan Wang, Mou Zeng, Xin Hao, Qi-hua He, Jing-pei 
Li, Xu-kai Li, Wei Wang, Li-min Ou, Ya-lei Zhang, Jing-wei Liu, Xin-guo Xiong, Wei-juna Shi, San-mei Yu, Run-dong Qin, Si-yang Yao, Bo-meng Zhang, Xiao-hong Xie, Zhan-hong Xie, Wan-di Wang, Xiao-xian Zhang, Hui-yin Xu, Zi-qing Zhou, Ying Jiang, Ni Liu, Jing-jing Yuan, Zheng Zhu, Jie-xia Zhang, Hong-hao Li, Wei-hua Huang, Lu-lin Wang, Jie-ying Li, Li-fen Gao, Jia-bo Gao, Cai-chen Li, Xue-wei Chen, Jia-bo Gao, Ming-shan Xue, Shou-xie Huang, Jia-man Tang, Wei-li Gu and Jin-lin Wang (Guangzhou Institute of Respiratory Health) for their dedication to data entry and verification. We are grateful to Tencent Co. Ltd. for their provision of the number of certified hospitals for admission of patients with Covid-19 throughout China. Finally, we thank all the patients who consented to donate their data for analysis and the medical staff working in the front line.

Author contributions: Wei-jie Guan, Wen-hua Liang, Jian-xing He, and Nan-shan Zhong participated in study design and study conception; Wen-hua Liang, Yi Zhao, Heng-rui Liang, Zi-sheng Chen, Chun-quan Ou, L. Li, Ping-yan Chen, Jian-fu Li, Cai-chen Li, Li-min Ou, Bo Cheng, Wei Wang and Shan Xiong performed data analysis; Ru-chong Chen, Chun-li Tang, Tao Wang, Ling Sang, Zheng-yi Ni, Jie Xiang, Yu Hu, L. Li, Hong Shan, Chun-liang Lei, Yi-xiang Peng, Li Wei, Yong Liu, Ya-hua Hu, Peng Peng, Jian-ming Wang, Ji-yang Liu, Zhong Chen, Gang Li, Zhi-jian Zheng, Shao-qin Qiu, Jie Luo, Chang-jiang Ye, Shao-yong Zhu, Lin-ling Cheng, Feng Ye, Shi-yue Li, Jin-ping Zheng, Nuo-fu Zhang, and Nan-shan Zhong recruited patients; Wei-jie Guan, Jian-xing He, Wen-hua Liang and Nan-shan Zhong drafted the manuscript; all authors provided critical review of the manuscript and approved the final draft for publication.

Support statement: Supported by National Health Commission, Department of Science and Technology of Guangdong Province. The funder had no role in the conduct of the study. Funding information for this article has been deposited with the Crossref Funder Registry.

Conflict of interest: Wei-jie Guan has nothing to disclose. Wen-hua Liang has nothing to disclose. Yi Zhao has nothing to disclose. Heng-rui Liang has nothing to disclose. Zi-sheng Chen has nothing to disclose. Yi-min Li has nothing to disclose. Xiao-qing Liu has nothing to disclose. Ru-chong Chen has nothing to disclose. Chun-li Tang has nothing to disclose. Tao Wang has nothing to disclose. Chun-quan Ou has nothing to disclose. Li has nothing to disclose. Ping-yan Chen has nothing to disclose. Ling Sang has nothing to disclose. Wei Wang has nothing to disclose. Jian-fu Li has nothing to disclose. Cai-chen $\mathrm{Li}$ has nothing to disclose. Li-min Ou has nothing to disclose. Bo Cheng has nothing to disclose. Shan Xiong has nothing to disclose. Zheng-yi Ni has nothing to disclose. Jie Xiang has nothing to disclose. Yu $\mathrm{Hu}$ has nothing to disclose. Lei Liu has nothing to disclose. Hong Shan has nothing to disclose. Chun-liang Lei has nothing to disclose. Yi-xiang Peng has nothing to disclose. Li Wei has nothing to disclose. Yong Liu has nothing to disclose. Ya-hua $\mathrm{Hu}$ has nothing to disclose. Peng has nothing to disclose. Jian-ming Wang has nothing to disclose. Ji-yang Liu has nothing to disclose. Zhong Chen has nothing to disclose. Gang Li has nothing to disclose. Zhi-jian Zheng has nothing to disclose. Shao-qin Qiu has nothing to disclose. Jie Luo has nothing to disclose. Chang-jiang Ye has nothing to disclose. Shao-yong Zhu has nothing to disclose. Lin-ling Cheng has nothing to disclose. Feng Ye has nothing to disclose. Shi-yue Li has nothing to disclose. Jin-ping Zheng has nothing to disclose. Nuo-fu Zhang has nothing to disclose. Nan-shan Zhong reports grants from the National Health Commission and Dept of Science and Technology of Guangdong Province, during the conduct of the study. Jian-xing He has nothing to disclose.

The version of this article originally published in early view format contained some transcription errors in the total subject numbers presented in table 3 . This was an issue that arose in compiling the table and the underlying results and conclusions are not affected by the correction.

\section{References}

World Health Organization. www.who.int Date last accessed: 10 March 2020.

2 World Health Organization. Coronavirus disease (COVID-19) situation reports. www.who.int/emergencies/ diseases/novel-coronavirus-2019/situation-reports/ Date last accessed: 10 March 2020.

3 Huang C, Wang Y, Li X, et al. Clinical features of patients with 2019 novel coronavirus in Wuhan, China. Lancet 2020; 395: 497-506.

4 Chen N, Zhou M, Dong X, et al. Epidemiological and clinical characteristics of 99 cases of 2019 novel coronavirus pneumonia in Wuhan, China: a descriptive study. Lancet 2020; 395: 507-513.

5 Wang $\mathrm{D}, \mathrm{Hu} \mathrm{B}, \mathrm{Hu} \mathrm{C}$, et al. Clinical characteristics of 138 hospitalized patients with 2019 novel coronavirus-infected pneumonia in Wuhan, China. JAMA 2020; in press [https://doi.org/10.1001/jama.2020.1585].

6 Kui L, Fang YY, Deng Y, et al. Clinical characteristics of novel coronavirus cases in tertiary hospitals in Hubei Province. Chin Med J 2020; in press [https://doi.org/10.1097/CM9.0000000000000744].

$7 \mathrm{Xu}$ XW, Wu XX, Jiang XG, et al. Clinical findings in a group of patients infected with the 2019 novel coronavirus (SARS-Cov-2) outside of Wuhan, China: retrospective case studies. BMJ 2020; 368: m606.

8 Chan JF, Yuan S, Kok KH, et al. A familial cluster of pneumonia associated with the 2019 novel coronavirus indicating person-to-person transmission: a study of a family cluster. Lancet 2020; 395: 514-523.

9 Zhang S, Li H, Huang S, et al. High-resolution CT features of 17 cases of coronavirus disease 2019 in Sichuan province, China. Eur Respir J 2020; in press [https://doi.org/10.1183/13993003.00334-2020].

10 Wang L, Gao YH, Iou L, et al. The clinical dynamics of 18 cases of COVID-19 outside of Wuhan, China. Eur Respir J 2020; in press [https://doi.org/10.1183/13993003.00398-2020].

11 Yao Y, Tian Y, Zhou J, et al. Epidemiological characteristics of SARS-CoV-2 infections in Shaanxi, China by 8 February 2020. Eur Respir J 2020; in press [https://doi.org/10.1183/13993003.00310-2020].

12 Guan WJ, Ni ZY, Hu Y, et al. Clinical characteristics of coronavirus disease 2019 in China. N Engl J Med 2020; in press [https://doi.org/10.1056/NEJMoa2002032].

13 Gao HN, Lu HZ, Cao B, et al. Clinical findings in 111 cases of influenza A (H7N9) virus infection. $N$ Engl J Med 2013; 368: 2277-2285.

14 Placzek HED, Madoff LC. Association of age and comorbidity on 2009 influenza A pandemic H1N1-related intensive care unit stay in Massachusetts. Am J Public Health 2014; 104: e118-e125.

15 Mauskopf J, Klesse M, Lee S, et al. The burden of influenza complications in different high-risk groups. J Med Economics 2013; 16: 264-277. 
16 Shiley KT, Nadolski G, Mickus T, et al. Differences in the epidemiological characteristics and clinical outcomes of pandemic (H1N1) 2009 influenza, compared with seasonal influenza. Infect Control Hosp Epidemiol 2010; 31: 676-682.

17 Martinez A, Soldevila N, Romeo-Tamarit A, et al. Risk factors associated with severe outcomes in adult hospitalized patients according to influenza type and subtype. PLoS One 2019; 14: 0210353.

18 Gutiérrez-González E, Cantero-Escribano JM, Redondo-Bravo L, et al. Effect of vaccination, comorbidities and age on mortality and severe disease associated with influenza during the season 2016-2017 in a Spanish tertiary hospital. J Infect Public Health 2019; 12: 486-491.

19 Booth CM, Matukas LM, Tomlinson GA, et al. Clinical features and short-term outcomes of 144 patients with SARS in the greater Toronto area. JAMA 2003; 289: 2801-2809.

20 Alqahtani FY, Aleanizy FS, Ali Hadi Mohammed R, et al. Prevalence of comorbidities in cases of Middle East respiratory syndrome coronavirus: a retrospective study. Epidemiol Infect 2018; 5: 1-5.

21 Badawi A, Ryoo SG. Prevalence of comorbidities in the Middle East respiratory syndrome coronavirus (MERS-CoV). Int J Infect Dis 2016; 49: 129-133.

22 Rahman A, Sarkar A. Risk factors for fatal Middle East Respiratory Syndrome coronavirus infections in Saudi Arabia: analysis of the WHO line list, 2013-2018. Am J Public Health 2019; 109: 1288-1293.

23 Alanazi KH, Abedi GR, Midgley CM, et al. Diabetes mellitus, hypertension, and death among 32 patients with MERS-CoV infection, Saudi Arabia. Emerg Infect Dis 2020; 26: 166-168.

24 Yang YM, Hsu CY, Lai CC, et al. Impact of comorbidity on fatality rate of patients with Middle East Respiratory Syndrome. Sci Rep 2017; 7: 11307.

25 Garbati MA, Fagbo SF, Fang VJ, et al. A comparative study of clinical presentation and risk factors for adverse outcome in patients hospitalised with acute respiratory disease due to MERS coronavirus or other causes. PLoS One 2016; 11: e0165978.

26 Rivers CM, Majumder MS, Lofgren ET. Risks of death and severe disease in patients with Middle East Respiratory Syndrome coronavirus, 2012-2015. Am J Epidemiol 2016; 184: 460-464.

27 Kulscar KA, Coleman CM, Beck S, et al. Comorbid diabetes results in immune dysregulation and enhanced disease severity following MERS-CoV infection. JCI Insight 2019; 20: e131774.

28 Matsuyama R, Nishiura H, Kutsuna S, et al. Clinical determinants of the severity of Middle East respiratory syndrome (MERS): a systematic review and meta-analysis. BMC Public Health 2016; 16: 1203.

29 World Health Organization. Clinical management of severe acute respiratory infection when COVID-19 is suspected. www.who.int/internal-publications-detail/clinical-management-of-severe-acute-respiratory-infectionwhen-novel-coronavirus-(ncov)-infection-is-suspected Date last updated: 13 March 2020; date last accessed: 10 March 2020.

30 Metlay JP, Waterer GW, Long AC, et al. Diagnosis and treatment of adults with community-acquired pneumonia: an official clinical practice guideline of the American Thoracic Society and Infectious Disease Society of America. Am J Respir Crit Care Med 2019; 200: e45-e67.

31 Li HY, Guo Q, Song WD, et al. Mortality among severe community-acquired pneumonia patients depends on combinations of 2007IDSA/ATS minor criteria. Int J Infect Dis 2015; 38: 141-145.

32 Gearhart AM, Furmanek S, English C, et al. Predicting the need for ICU admission in community-acquired pneumonia. Respir Med 2019; 155: 61-65.

33 Fang L, Gao P, Bao H, et al. Chronic obstructive pulmonary disease in China: a nationwide prevalence study. Lancet Respir Med 2018; 6: 421-430.

34 Moni MA, Lionel P. Network-based analysis of comorbidities risk during an infection: SARS and HIV case studies. BMC Bioinformatics 2014; 15: 333.

35 Naqvi AA, Shah A, Ahmad R, et al. Developing an integrated treatment pathway for a post-coronary artery bypass grating $(\mathrm{CABG})$ geriatric patient with comorbid hypertension and type 1 diabetes mellitus for treating acute hypoglycemia and electrolyte imbalance. J Pharm Bioallied Sci 2017; 9: 216-220.

36 Murphy TE, McAvay GJ, Allore HG, et al. Contributions of COPD, asthma, and ten comorbid conditions to health care utilization and patient-centered outcomes among US adults with obstructive airway disease. Int $J$ Chron Obstruct Pulmon Dis 2017; 12: 2515-2522. 